\title{
CRISIS POLÍTICA Y TRANSICIONES EN VENEZUELA
}

\author{
POLITICAL CRISIS AND TRANSITIONS IN VENEZUELA
}

\author{
Carlos A. Romero $\left.{ }^{*}\right)$ \\ Universidad Central de Venezuela, Caracas, Venezuela
}

Resumen: Venezuela ha conocido recientemente tres transiciones. La primera transcurrió entre la caída del dictador Marcos Pérez Jiménez y el desarrollo de un modelo de democracia representativa. La segunda transcurrió entre la llegada de Higo Chávez a la presidencia y su posterior fallecimiento. La tercera se desarrolla desde la muerte física de Chávez hasta la presidencia de su sucesor Nicolás Maduro. Este artículo analiza el desenvolvimiento de esas tres etapas, los actores que participan en ellas, las políticas públicas generadas así como su entorno social y económico, haciendo un énfasis en la situación actual y en sus proyecciones.

Palabras claves: Venezuela; Chávez; Maduro.

Abstract: Venezuela has recently seen three transitions. The first occurred between the fall of the strongman Marcos Perez Jimenez and the development of a model of representative democracy. The second elapsed between the arrival of Hugo Chávez to the presidency and his death. The third is developed from the death of Chávez to the presidency of his successor Nicolás Maduro. This article analyzes the development of these three stages, the actors involved in them, the government public policies and their social and economic environment, with an emphasis on the current situation and its projections.

Keywords: Venezuela; Chávez; Maduro.

(*) Doctor en Ciencias Políticas. Profesor del Instituto de Estudios Políticos de la Universidad Central de Venezuela. E-mail: <romecan53@hotmail.com>. Recibido en: 3.12.2015, aceptado en: 29.2.2016. 


\section{INTRODUCCIÓN}

Pertenezco a una generación de politólogos venezolanos a quienes les enseñaron a comprender la política nacional con base en un enfoque considerado como el "paradigma" a seguir en la década de los setenta. Me refiero al llamado "Sistema Populista de Conciliación de Élites" diseñado por el reconocido profesor e investigador Juan Carlos Rey Martínez (REY, 1972; REY, 1989; REY, 1991).

Según este autor, el sistema político venezolano estaba fundamentado en la presencia de dos partidos democráticos mayoritarios, Acción Democrática, AD (socialdemócrata) y COPEI (socialcristiano), un fuerte respaldo económico por parte de un Estado petrolero, una economía rentista, la neutralidad de las Fuerzas Armadas, un reconocimiento internacional a la democracia y un apoyo mayoritario social, tanto del sector empresarial como de las clases medias y los sectores obreros y campesinos (REY, 1972; LEVINE, 1973; KORNBLITH, 2007).

Este enfoque no obtuvo el apoyo unánime de todos los sectores políticos y académicos venezolanos. Desde la izquierda, hubo quienes alertaron que el modelo venezolano no era más que otro ejemplo latinoamericano del reformismo y que en verdad lo que se quería construir era un modelo que ocultara la dependencia del país del capitalismo internacional, especialmente de Estados Unidos. Desde la óptica de algunos sectores conservadores, se estimaba que Venezuela no cumplía con las condiciones mínimas para desarrollar el modelo democrático y que por lo tanto se requería de un gobierno autoritario para lograr el desarrollo. Finalmente, estaban aquellos seguidores del pensamiento liberal quienes propugnaban un régimen de libre empresa para Venezuela y la reducción del peso del populismo y del Estado (ROMERO, 2011; SILVA, 1967; NAIM y PIÑANGO, 1984; ROMERO, 1986).

La coexistencia de esos diversos enfoques originó un importante debate en Venezuela, entre los analistas radicados en el país y los extranjeros que estudiaron la experiencia venezolana a la luz de los debates teóricos imperantes en las décadas de los sesenta y setenta del siglo XX.

Cabe recordar el aporte pionero de los politólogos estadounidenses Robert Alexander, John Martz, David Myers y Daniel Levine, quienes enfocaron sus estudios sobre los partidos políticos y las elecciones en Venezuela. Levine fue más allá de esos temas, en función de analizar cómo se compartía el poder político en el país, utilizando la literatura que enfatiza las relaciones corporativistas entre los actores principales y cómo se regulaban el cambio político y los conflictos entre ellos de una manera colegiada (LEVINE, 1973; MARTZ y MYERS, 1977; MC COY y MYERS, 2004; BOND, 1977).

El enfoque predominante ya citado definió a Venezuela como un caso "sui-generis" dadas las negociaciones entre los partidos y otros sectores importantes de la vida nacional, a fin de lograr un consenso sobre el carácter democrático del Sistema de Conciliación de Élites, la existencia de una nueva constitución, como fue la Constitución de 1961, el respeto a la autonomía de los poderes públicos y la convocatoria a elecciones periódicas (REY, 1972; ELLNER, 1977). 
En el mundo y en América Latina se dieron previamente otros modelos parecidos a fin de establecer un juego democrático, tal como fue el caso de Noruega en la postguerra y el caso de Colombia, apenas unos meses anteriores a la caída del dictador venezolano Marcos Pérez Jiménez (el 23 de enero de 1958). El modelo venezolano se concretó con la firma del Pacto de Punto Fijo (de donde se deriva el vocablo "puntofijismo") en el mes de octubre de 1958, un acuerdo firmado por los representantes de los partidos políticos AD, COPEI y Unión Republicana Democrática (URD), quienes se comprometieron a apoyar al candidato ganador en las elecciones presidenciales de diciembre de ese mismo año (REY, 1972; LEVINE, 1973).

El período histórico que transcurrió durante esos 41 años, de 1958 a 1999 presentó diversas características y generó múltiples interpretaciones y no es nada extraño que provoquen aún en nuestros días un interesante debate. La tesis con mayor apoyo es la que plantea que la estabilidad política alcanzada en Venezuela de 1958 en adelante se debió a la conjunción de dos factores determinantes: el régimen de partidos democráticos marcado por el bipartidismo de AD y de COPEI, con el concurso de otros partidos minoritarios y los ingresos petroleros que hicieron de la economía venezolana un modelo basado en el rentismo con el papel predominante del Estado (LEVINE, 1973; CARRERA, 1977; BAPTISTA, 1997; ÁLVAREZ, 2000; DUNNING 2008).

El "Sistema Populista de Conciliación de Élites", conocido coloquialmente como el "Pacto de Punto Fijo" y también como el "puntofijismo" no tuvo (y no tenía por qué tenerlo) un desarrollo lineal sin la presencia de las disfunciones y críticas que caracterizan a cualquier proceso político. En primer lugar, el régimen tuvo que enfrentarse a cinco movimientos insurgentes en el seno de las Fuerzas Armadas. Nos referimos a las tres sublevaciones de carácter conservador del general Jesús María Castro León (1958 y 1960) y en la ciudad de Barcelona en 1960 (conocido como "El Barcelonazo") y de los alzamientos de izquierda ocurridos en las ciudades de Carúpano y Puerto Cabello en 1962 (conocidos como "El Carupanazo" y "el Porteñazo" respectivamente), todos ellos dirigidos a derrocar al naciente régimen democrático (ROMERO, 2006; KORNBLITH, 1997; LEVINE, 1973).

En segundo lugar, el régimen tuvo que enfrentar la lucha armada, entre los años de 1960 a 1980 producto de la decisión de algunos sectores de izquierda radicados en el seno de Acción Democrática (AD), del Partido Comunista de Venezuela (PCV), en Unión Republicana Democrática (URD) y dentro de las Fuerzas Armadas y sectores sindicales, intelectuales y estudiantiles que como un todo se distanciaron del Pacto de Punto Fijo y se abocaron a tratar de implantar en Venezuela una revolución parecida a la cubana (CHILCOTE, 1970; ROMERO, 2011; CHALIAND, 2008).

Reducida la lucha armada en Venezuela a fines de los años sesenta, la mayoría de los sectores críticos que se habían levantado en contra del régimen canalizaron sus demandas dentro del marco de la lucha pacífica y la competencia electoral. Un grupo reducido continuó en el camino de la lucha armada y obtuvo de nuevo el apoyo de la Cuba revolucionaria (ROMERO, 2011).

En tercer lugar, esos sectores críticos, incluyendo aquellos que no se fueron a la lucha armada y que continuaron participando en la vida legal, evaluaron negativamente 
al régimen de partidos en Venezuela, enfatizando la poca eficacia observada para hacer cumplir las promesas que los partidos mayoritarios habían ofertado en sus sucesivas campañas electorales (KARL, 1997).

De una manera u otra, algunos partidos políticos, los disidentes de los partidos políticos mayoritarios, las personalidades críticas y otros actores sociales se definieron a sí mismos como los "excluidos" del Pacto de Punto Fijo, incluyendo a los seguidores del ex-dictador Marcos Pérez Jiménez. Esto contó con los intentos de configurar unas candidaturas presidenciales de izquierda distintas al "puntofijismo" en las elecciones presidenciales, parlamentarias y municipales y las importantes candidaturas presidenciales y conservadoras del escritor Arturo Uslar Pietri en 1963, la del propio Pérez Jiménez para 1973 y la del conocido locutor de televisión Renny Ottolina en 1978. Estos dos últimos intentos no se concretaron; el primero por la enmienda constitucional de 1969 que le prohibió a Pérez Jiménez asumir su curul de senador (obtenida en diciembre de 1968) y aspirar a otro cargo público, incluyendo la presidencia de Venezuela y el segundo, por que Ottolina murió en un accidente de aviación en plena campaña electoral (KORNBLITH, 1997; NAIM y PIÑANGO, 1984; MC COY y MYERS, 2004; BAPTISTA, 1997; BLANCO, 1993).

En cuanto a la política exterior, los gobiernos venezolanos desarrollaron una diplomacia moderada entre 1958 y 1999 en donde sólo en momentos críticos internacionales tomaron una posición definida. Por una parte, Venezuela buscó tener una relación especial con Estados Unidos en donde se caracterizó al país como un aliado seguro y confiable en el marco del envío de más del 50\% de la producción petrolera venezolana al mercado estadounidense. En segundo lugar, Venezuela procuró en el ámbito latinoamericano y caribeño promover el sistema democrático y la integración económica. En tercer lugar, Caracas buscó resolver los problemas limítrofes sostenidos con Colombia y con Guyana dentro de un proceso de negociación pacífica con esos países. Con Colombia, en referencia a la delimitación de áreas marinas y submarinas al norte del Golfo de Venezuela y en cuanto a Guyana, en torno a la justa reclamación de Venezuela de parte del territorio guyanés conocido como el territorio Esequibo en el marco del Acuerdo de Ginebra, instrumento jurídico firmado en el año 1966 por el Reino Unido, Venezuela y la entonces naciente república de Guyana. En cuarto lugar, promover una política exterior más activa en la procura de un orden mundial más equitativo (CARDOZO, 1998; ROMERO, 2006; BOND, 1977).

En este contexto, Venezuela fue - entre muchas iniciativas llevadas a cabo durante esos años - un importante miembro de la Organización de Estados Americanos (OEA) en donde defendió la democracia representativa frente a las dictaduras latinoamericanas y acusó a Cuba de injerencia en los asuntos internos del país, en tanto que fue promotora y fundadora de la Organización de Países Exportadores de Petróleo (OPEP) en 1960, promovió en el seno de las Naciones Unidas el diálogo Norte-Sur, impulsó los acuerdos de integración de la ALADI y del Acuerdo de Cartagena, conocido más tarde como la Comunidad Andina de Naciones (CAN), así como el Grupo de los Tres junto con Colombia y México, promovió los acuerdos de paz en Centroamérica junto a México y otros países, a través del Grupo de Contadora y estimuló el diálogo regional con la Comunidad Europea (conocida más tarde como la Unión Europea). Además, Venezuela fue uno de 
los primeros países latinoamericanos que se acercó a los países caribeños, asiáticos y africanos (CARDOZO, 1998; ROMERO, C, 2006; BOND, 1977).

Cabe destacar que en este proceso diplomático e integracionista hubo importantes contratiempos, tanto internos como externos. La política exterior de Venezuela hacia Estados Unidos, Cuba, los temas fronterizos, la política petrolera y la integración económica no obtuvieron el apoyo unánime de los venezolanos. Hubo importantes críticas de sectores empresariales a esas iniciativas, tales como el rechazo de buena parte del empresariado privado a la incorporación de Venezuela a la OPEP y a la Junta de Cartagena en 1969; de sectores militares a las negociaciones territoriales con Colombia y con Guyana y algunas diferencias de los gobiernos de la época con sectores políticos internos, en cuanto a las políticas de asociación con Estados Unidos y de deslinde con Cuba (ROMERO, 2011; NAIM y PIÑANGO, 1984; MARTZ y MYERS, 1977).

De igual modo, Venezuela tuvo unos importantes desencuentros diplomáticos con Estados Unidos en relación a la creación de la OPEP, a la falta de apoyo de Washington a la promoción de la democracia en la región, a la invasión estadounidense a la República Dominicana en 1965, sobre la política de Estados Unidos hacia Nicaragua en los años ochenta, sobre el apoyo de Estados Unidos al Reino Unido en la Guerra de las Malvinas en 1982 y sobre los alcances de la política comercial hemisférica en la década de los noventa (MC COY y MYERS, 2004).

Así mismo, Venezuela sostuvo entre 1960 y 1974 unas importantes diferencias políticas e ideológicas con Cuba y ciclos negativos con Colombia, dado el cúmulo de tensiones fronterizas entre las dos naciones y relacionadas con contrabando, narcotráfico, movimientos armados, migraciones y la falta de un acuerdo sobre la delimitación de áreas marinas y submarinas (BOND, 1977; ROMERO, 2011).

Para la década de los ochenta del siglo XX, Venezuela entró a una nueva etapa en la cual la crítica al sistema político no fue la exclusiva de los sectores insurgentes o ideológicos que se habían declarado tempranamente en contra del "puntofijismo", sino más bien se extendió hacia otros sectores que habían apoyado al régimen y que ahora estaban en contra de los partidos mayoritarios y pedían una apertura política y una mayor equidad social. Al decir de un importante líder político de entonces, el pueblo estaba "bravo" y reclamaba la concreción de las promesas electorales de unos partidos que estaban perdiendo vertiginosamente su respaldo popular (NAIM y PIÑANGO, 1984; ROMERO, 1986).

Tres momentos históricos se convirtieron en los símbolos de ese movimiento crítico. Por una parte el hecho de la devaluación oficial del bolívar en 1983 frente al dólar, luego de muchos años con una tasa fija y con plena libertad de cambios, lo que llevó a la generación del fenómeno de la inflación y de una crisis cambiaria poco conocida en Venezuela. Por la otra, nadie predijo con exactitud la insurgencia social del 27 y 28 de febrero de 1989 conocida como el "Caracazo", hecho social que sorprendió a los que todavía creían que Venezuela era un modelo estable que contaba con un amplio respaldo popular. Y en tercer lugar, ocurrió lo que pocos autores advirtieron, una insurrección militar (KORNBLITH, 2007). 
En las Fuerzas Armadas de Venezuela se había desarrollado un grupo importante de oficiales y tropas fuertemente críticos del "puntofijismo" que se rebelaron en dos ocasiones en contra del presidente Carlos Andrés Pérez, en febrero y en noviembre de 1992. Estos dos intentos de golpe de Estado fracasaron en cuanto al objetivo de la toma del poder, pero generaron un fuerte y controversial impacto en la vida política de Venezuela y en el resto de América Latina (DIAMINT, 2005).

En este contexto, el "Viernes negro", tal como se conoció el día que se devaluó la moneda por primera vez en muchos años, en 1983; el "Caracazo" en 1989 y las rebeliones militares de 1992, fueron los puntos visibles de una crisis generalizada del régimen de partidos conocido como el "puntofijismo", crisis que duró varios años y en donde se dieron muchas contradicciones y disensos entre los actores políticos: los que querían salvaguardar lo que se había acumulado en términos democráticos y los que propugnaban un cambio de régimen (KORNBLITH, 1997; KORNBLITH, 2007).

El ex-presidente Rafael Caldera entendió muy bien en 1992 ese deseo de cambio y ofreció su liderazgo para recuperar el régimen y no cambiarlo. Caldera creyó que el problema estaba radicado en la degeneración del sistema político por la corrupción administrativa y por la ilegitimidad del régimen y que volviendo a los orígenes del proceso este podía salvarse. Pero él no pudo hacerlo y su gobierno (1994-1999) no pudo detener la ruptura de las élites y el movimiento de masas crítico que buscaba algo más que regenerar el sistema (CORRALES y PENFOLD, 2011).

Hugo Chávez, el líder militar más importante de las rebeliones de 1992, encarnó el deseo de cambio de los inconformes con el régimen democrático y a su vez reflejó el deseo de transformar la sociedad venezolana, de apostar por algo nuevo, en medio de la pérdida de consenso, de una severa crisis económica producto de la baja de los precios del petróleo, de la crisis cambiaria, del efecto desestabilizador de la deuda externa y de la ineficiencia estatal. Chávez ganó las elecciones presidenciales de 1999 y se abrió un nuevo capítulo en la historia del país (DUNNING, 2008; SMILDE y HELLINGER, 2011).

Con base en las consideraciones anteriores, el objetivo de este artículo es el de propiciar un análisis ponderado de la gestión pública durante los gobiernos de Hugo Chávez (1999-2013) y muy especialmente de Nicolás Maduro (2013) sobre una hipótesis de trabajo que plantea que en este momento se está observando una "crisis sin solución" en el régimen que se inició en 1999.

Se sostiene el argumento que a pesar de la desaparición física de Hugo Chávez en 2013 y de la actual crisis económica del país, alarmante desde el año 2014, el régimen no necesariamente está en su fase terminal. Todavía los actores que manejan al país desde 1999 tienen suficientes recursos materiales e inmateriales para sostenerse en el poder, a pesar del auge de la oposición, de una creciente crítica popular al régimen y de la propia metamorfosis del régimen hacia un camino autoritario (WEYLAND, 2003; WEYLAND, 2009).

Aparte de esta introducción dedicada a plantear unos antecedentes generales, en la segunda sección de este artículo se analiza la manera cómo se dio la transición en Venezuela de un régimen democrático reformista a un régimen revolucionario con una clara 
tendencia iliberal. En la tercera sección se estudia el comportamiento del gobierno del presidente Maduro a la luz de sus específicas condiciones externas e internas, haciendo énfasis en la coyuntura actual. En la cuarta sección se esbozan algunas conclusiones, se aportan algunas conjeturas y se proyectan algunas tendencias sobre el futuro cercano de Venezuela.

\section{UNA TRANSICIÓN PREVISTA}

Hugo Chávez ganó las elecciones presidenciales en Venezuela en diciembre de 1999 con el 56\% de los votos válidos. En dicha ocasión, la mayoría de los analistas y periodistas especializados plantearon que se estaba dando un "parte aguas" en la vida política del país. En efecto, la coalición político-electoral que se había formado alrededor del carismático líder de las asonadas militares de 1992 y que ganó, tanto las elecciones parlamentarias de septiembre de 1999 como las elecciones presidenciales de diciembre de 1999, era una alianza heterogenia formada por diversos partidos, dirigentes políticos y grupos sociales, entre los cuales estaba una importante representación de oficiales de las Fuerzas Armadas en situación de retiro (CORRALES y PENFOLD, 2011; DUNNING, 2008; SMILDE y HELLINGER, 2011).

En el marco de esa alianza destacaron esos oficiales retirados encabezados por Hugo Chávez, a quien se le había otorgado el sobreseimiento de la causa que lo tenía detenido, a través de una polémica decisión del presidente Caldera en 1994. Chávez y otros miembros del grupo insurreccional de 1992, habían decidido participar en las elecciones de 1998 luego de tener al menos hasta finales del año 1995 muchas reservas sobre la pertinencia del camino electoral para la toma del poder (CORRALES y PENFOLD 2011; CORRALES y ROMERO, 2013).

De alguna manera el país estaba preparado para una victoria del "chavismo", tal como se fue conociendo al movimiento disidente encarnado por el líder carismático. AD y COPEI habían escogido respectivamente a dos candidatos presidenciales con bastantes limitaciones personales, el dirigente político Luis Alfaro Ucero y la ex-reina de belleza Irene Sáez; a su vez, el candidato "outsider" de origen empresarial (Henrique Salas Romer) no logró remontar las encuestas y el gobierno de Caldera no apoyó a ningún candidato. A esto hay que agregar la situación general del país evaluada negativamente por la mayoría de los votantes que sufragaron por Hugo Chávez y en menor medida por Salas Romer. Este último recibió al final del proceso el apoyo de AD y de COPEI en un intento desesperado de esos partidos de evitar la victoria de Chávez (GÓMEZ y PATRUYO, 2000; ROSS, 2011; MORGAN, 2011).

Desde un primer momento, la coalición electoral que llevó al "chavismo" al poder giró en torno a un programa político y económico orientado por tres objetivos principales, lo cual de alguna forma corresponde a tres variables típicas de los procesos revolucionarios. En primer lugar, se trataba de un grupo de líderes militares y civiles que por edad, procedencia social y profesional y su visión del mundo significó para la historia de Venezuela un desplazamiento de élites, cuyos simpatizantes fueron ocupando los poderes públicos al igual que la Administración Pública, las Fuerzas Armadas y la empresa 
estatal Petróleos de Venezuela - PDVSA (MC COY y MYERS, 2004; LEVITZKY y WAY, 2010; TAGLIEFF, 2007; WEYLAND, 2003; WEYLAND, 2009).

En segundo lugar, los nuevos gobernantes rompieron con el pasado, diseñando una exitosa campaña mediática que colocaba en sus hombros la representación de algo cronológicamente diferente, acuñando así los conceptos de la "Cuarta" y de la "Quinta República". La "Cuarta República" asignada a observar negativamente el período de la democracia representativa (1958-1999) y la Quinta República utilizada para observar el naciente régimen (KORNBLITH, 2006; DIETZ y MYERS, 2007).

En tercer lugar, diversos analistas, académicos y políticos venezolanos y extranjeros comprendieron que esa dirección política que desplazó a lo anterior y que rompía con el pasado tenía también un programa político que en los primeros años de su gestión fue definido como de izquierda y en el marco de la irrupción de gobiernos similares en América Latina a comienzos del siglo XXI (MC COY y MYERS, 2004; LINDBERG, 2009).

Desde un primer momento comenzó el debate sobre cómo clasificar el caso venezolano a la luz de la política comparada. Para algunos autores el chavismo correspondía a las características generales del populismo latinoamericano y para otros no era más que el ejemplo de un gobierno autoritario que comenzaba a desmontar la experiencia democrática venezolana. Para el resto, era un fenómeno revolucionario con características radicales (CORRALES, 2006; DIETZ y MYERS, 2007; HACHEMAOUI, 2012; COPPEDGE, 2005; MAINWARING, 2012).

Con la victoria de Chávez en 1998 quien ganó las elecciones con el 56.20\% de los votos, con el ejercicio de su presidencia desde 1999 y con la convocatoria inmediata a un proceso constituyente, la promulgación de la nueva Constitución de 1999 y la realización de las siguientes elecciones presidenciales en el año 2000 (en donde Chávez volvió a ganar con $59.76 \%$ de los votos), se sembraron las bases de lo que va a ser una de las más controversiales experiencias latinoamericanas del siglo XXI.

Entre 1999 y el año 2002, se fue perfilando un modelo político "reformista" que tuvo como características principales el liderazgo carismático de Chávez, el apoyo mayoritario de las masas populares, la intención de controlar por medio del Estado la economía, el comercio exterior, la política fiscal y la política cambiaria y la des-institucionalización de las Fuerzas Armadas, de PDVSA y de otras empresas públicas (CORRALES y ROMERO, 2013; MAINWARING, 2012).

El país no chavista respondió a estas decisiones "gota en gota". Por una parte, una representación del sector empresarial, del sector militar y parte del sector sindical, más sectores de la clase media profesional que habían apoyado a Chávez en 1999 y con menor fuerza en el año 2000, comenzaron a retirarle su apoyo. Esto fue seguido por la generación de un movimiento de la sociedad civil venezolana que comenzó a comprender que el chavismo era algo más que un gobierno de izquierda democrática y que en verdad se estaban echando las bases de un régimen que tenía una fuerte orientación iliberal (COPPEDGE, 2005).

Este movimiento crítico llevó a una serie de manifestaciones políticas de carácter social y a la extensión de las reservas que desde 1992 tuvieron los dirigentes políticos 
de los partidos democráticos sobre los líderes del nuevo régimen. La oposición venezolana que había quedado fracturada con las victorias presidenciales de Hugo Chávez y el triunfo del chavismo en una serie de otras elecciones pudo remontar el camino opositor a lo que ya se observaba como la pretensión de aplicar un modelo político excluyente y hegemónico, a pesar que casi la mitad de los venezolanos se oponían al nuevo régimen (MARTÍNEZ, 2012).

Entre el año 2001 y 2003, Venezuela asistió a un duelo sobre el poder. El gobierno y el oficialismo estaban interesados en profundizar su modelo y la oposición en rechazarlo. Tres acontecimientos históricos van a cambiar esa correlación cuasi-paritaria a favor del chavismo. Por una parte, las huelgas, paros laborales y empresariales y manifestaciones de la oposición y del empresariado y del sector sindical marcaron un momento en el cual parte de la oposición venezolana se definió por un cambio de régimen. Esto dio lugar a un clima insurreccional que explotó en abril del año 2002 con un acontecimiento singular: un grupo de militares y civiles opositores tomaron el poder en Venezuela por espacio de 48 horas y colocaron al dirigente empresarial Pedro Carmona como el presidente provisional del país. Chávez salió del poder y estuvo detenido y custodiado por parte del sector militar insurrecto (MARTÍNEZ, 2012; ROMERO, 2006).

Pero otro sector castrense se negó a secundar esa acción y presionó para el regreso de Chávez a la presidencia y la restauración del régimen chavista. Sin el respaldo mayoritario de las Fuerzas Armadas, sin el apoyo popular que en su mayoría reclamaba el retorno de Chávez al poder, sin un apoyo certero de la comunidad internacional que definió de manera casi unánime todo este proceso como anti-constitucional, más la existencia de fricciones internas dentro del grupo insurreccional; todo ello determinó que la rebelión cívico-militar de abril de 2002 durara muy poco tiempo en el poder (DIAMINT, 2005).

Sin embargo, el retorno del chavismo al poder, luego de ese corto intermedio se convirtió en una lección para el chavismo. Desde un primer momento, a Chávez y a sus seguidores les quedó claro que era necesario un "revolcón" en la estructura del poder en Venezuela. El primer paso fue aprovechar la coyuntura vivida y la división del liderazgo militar para definir de una vez por todas, que las Fuerzas Armadas no debían ser neutrales ni mucho menos opositoras al gobierno de Chávez. El proceso de depuración en el seno de esa institución fue directo y concreto, no sólo en términos personales en contra de los oficiales y tropas comprometidos con "el Carmonazo" (tal como se conoció el golpe de Estado de abril de 2002), sino en términos de una doctrina militar diferente, una politización permanente de la institución y un control interno. El segundo paso fue el de controlar a PDVSA y a su personal profesional y obrero (MAINWARING, 2012; MARTÍNEZ, 2012).

Luego de una huelga petrolera convocada por sectores profesionales y laborales dentro de PDVSA en diciembre de 2002, de un sucesivo proceso de cambios en la directiva de la empresa y de la suspensión de actividades por parte de algunos profesionales y técnicos, se produjo la semi-paralización de la industria petrolera en Venezuela, en cuanto a las tareas de explotación, refinación, transporte, mercadeo y actividades conexas, al igual que en el sector gasífero. Al concluir la huelga petrolera en febrero de 2003 y con 
la expulsión de la empresa de alredor de 18 mil empleados, el gobierno retomó el control absoluto de PDVSA y a su vez acabó con su perfil neutral-gerencial convirtiéndola en una pieza más en el proceso de control del sector público en Venezuela por parte de los nuevos gobernantes (DIAMINT, 2005; ROMERO, 2006).

Cabe destacar el importante rol que cumplieron la Organización de Estados Americanos (OEA), el Centro Carter y las Naciones Unidas, a través del Programa para el Desarrollo (PNUD) en fomentar un diálogo entre el gobierno y la oposición durante los años 2003 y 2004, diálogo que se plasmó en la formación de una mesa de negociación y acuerdos que trató de buscar una salida pacífica y democrática a la crisis venezolana y que desembocó en la convocatoria de un referéndum revocatorio presidencial en el año 2004 (MARTÍNEZ, 2012).

El control de las Fuerzas Armadas y de PDVSA no significó que el gobierno tuviera las manos libres para ejercer la hegemonía que deseaba implantar. Los sectores críticos en Venezuela contaron con el respaldo de una parte de la comunidad internacional a fin de advertir el eclipse democrático de un país que se encaminaba hacia un sendero revolucionario y excluyente. Por primera vez, las encuestas de opinión indicaban que en los sectores populares estaban germinando significativamente grupos críticos a la labor del gobierno, en la medida en que las cifras y los especialistas encargados de estudiarlas expresaban sendos déficits en el área socio-económica del país, en términos de desempleo e inflación y sobre todo en algunas fallas en los programas sociales (MAINWARING, 2012).

El gobierno y el partido de gobierno, el hasta entonces Movimiento V República (MVR) (Cambiará su nombre y su estructura en el año 2006 para llamarse desde entonces el "Partido Socialista Unificado de Venezuela", PSUV) crearon en el año 2003 el sistema de las "misiones sociales" dirigidas a solventar los problemas más urgentes de la población; salud, educación, vivienda, empleo, en el marco de una cooperación estratégica con el gobierno de Cuba. El lanzamiento de estas misiones vino ligado a la convocatoria de un referéndum revocatorio del mandato del presidente Chávez realizado en agosto de 2004, el cual fue ganado por quienes dijeron que "no" a la salida constitucional del presidente Chávez, con un 59.1 \% de los votos (ROMERO, 2006).

En el año 2005, la oposición partidista decidió no participar en las elecciones parlamentarias y de hecho le dio una "carta blanca" al oficialismo que ocupó todas las curules para ese período parlamentario hasta el año 2010. (Algunos diputados elegidos en las listas oficialistas rompieron más tarde con el chavismo y se formó una pequeña fracción parlamentaria disidente del régimen) (DIETZ y MYERS, 2007).

En el año 2006 se realizaron unas nuevas elecciones presidenciales en donde compitieron el propio presidente Chávez para reelegirse por segunda vez y para comenzar su tercer período presidencial y el candidato unitario de la oposición, el entonces gobernador del estado Zulia Manuel Rosales. Chávez obtuvo la victoria con el $62.84 \%$ de los votos y Rosales obtuvo un 36.90\% de los votos.

La elecciones de 2006 demostraron dos cosas. En primer lugar que el régimen se había consolidado y que durante la campaña electoral, el chavismo había perfilado y 
extendido su doctrina socialista con la bandera del "socialismo del Siglo XXI". Y en segundo lugar, que a pesar de tantas circunstancias adversas, la oposición mantenía una presencia electoral, social y mediática, en el marco de una polarización de la sociedad venezolana (DIETZ y MYERS, 2007).

En el año 2007 se convocó a un referéndum constitucional a fin de aprobar o no una serie de reformas constitucionales tendientes a abrir la oportunidad de la reelección presidencial indefinida y que en términos sencillos no fue más que el mecanismo central para el perfeccionamiento del control político del gobierno y del oficialismo sobre el país. Ese referéndum lo ganó la oposición por un escaso margen, 51\% a 49\% pero suficiente para que al gobierno no le quedara más remedio que manipular por la vía del Tribunal Supremo de Justicia la aprobación de todas las propuestas establecidas en la consulta (DUNNING, 2008).

Pero esto no es lo más importante. Se trataba del controlar el Poder Judicial para conseguir lo que buscaban el gobierno y el partido de gobierno: perfeccionar el control del poder Ejecutivo sobre el resto de los poderes públicos con el fin de profundizar no sólo el socialismo del Siglo XXI, sino la puesta en marcha de un programa de nacionalizaciones y privatizaciones de empresas privadas y la creación de un Estado empresario paralelo y de un sector privado afecto al gobierno que se conocerá más tarde con el nombre de los "Boliburgueses" (CORRALES y PENFOLD, 2011).

En el año 2010 se celebraron las elecciones parlamentarias para el período 20112016, en donde la oposición obtuvo una importante votación, 47\% que no se refleja en el número de curules que favoreció al gobierno y al partido de gobierno (98 a 65). Pero esto no es lo más importante, sino que en materia del funcionamiento del nuevo período parlamentario los diputados de la bancada oficialista se comportan como si tuvieran la mayoría absoluta y no le reconocieron a la oposición su derecho a estar en la directiva de la Asamblea y de obtener algunas presidencias de las comisiones permanentes parlamentarias.

Los analistas de la situación venezolana no se sorprendieron de esta manipulación y más bien advirtieron que para ese entonces se había consolidado no sólo un régimen, sino sobre todo una manera de gobernar imperativa. En efecto, los fenómenos políticos del sectarismo y del ventajismo estaban también perfeccionándose, no sólo en el control de los poderes públicos, lo que llevó a cuestionar el principio de su respectivas autonomías, sino también en cuanto a la creciente compra por la vía directa del Estado o por la vía indirecta, a través de grupos afines al régimen, de plantas de televisión, estaciones de radio, páginas web, periódicos y otros medios de comunicación, dentro de una campaña mediática tendiente a reproducir narrativas, mensajes, imágenes y perspectivas afines al régimen.

Las limitaciones a la democracia, la reducción del espacio doméstico de la oposición, el control mediático y la toma del control de las instituciones públicas dieron lugar a la plena consolidación del régimen (TAGLIEFF, 2007; WEYLAND, 2009; CORRALES y PENFOLD, 2011).

La política exterior de Venezuela significó también una palanca fundamental en esta transición. Desde 1999 Venezuela es un país con una política exterior revisionista. 
Esta conducta se observa en el tratamiento del tema de los derechos humanos, la oposición a la tesis de la ONU del "derecho a proteger", la crisis del mundo árabe, la incursión de Estados Unidos en Irak, de la OTAN en Libia, en la presión sobre Siria, en su desacuerdo sobre las sanciones de Naciones Unidas en contra de Irán y Rusia, en relación al tratamiento político que se le debe dar a Estados Unidos, en la visión venezolana del socialismo del siglo XXI, en las diferencias de Venezuela sobre el tema del combate al narcotráfico y en relación al tema de la presencia guerrillera en Colombia (CORRALES y ROMERO, 2013; TAGLIEFF, 2007; WEYLAND, 2009; CORRALES y PENFOLD, 2011).

A principios del siglo XXI se abrió nuevamente un ciclo de gobiernos izquierdistas en la región. El eje anti-imperialista y anti-estadounidense y una política a favor de Cuba jugaron desde entonces un papel estelar en la creación y en el impulso de las políticas domésticas y exteriores de esos gobiernos. Venezuela es uno de ellos (CORRALES y ROMERO, 2013).

Venezuela ha ofertado ese "paquete ideológico" basado en la tesis del "socialismo del Siglo XXI", que ofrece un modelo revolucionario con el papel predominante del Estado, la organización descentralizada del poder político, la reelección indefinida presidencial y la inclusión de los sectores desposeídos, dentro de varias formas de organización social y bajo la "sombrilla" del llamado poder comunal.

La política exterior bolivariana y la seguridad y defensa se fundamenta en la tesis de la guerra asimétrica, entendida esta como la puesta en práctica de una política defensiva social-militar del país ante cualquier agresión que se califique como tal, dentro de la idea de una supuesta correlación entre el enemigo externo y el enemigo interno (CORRALES y ROMERO, 2013).

La mezcla de unas raíces ideológicas marxistas, de tradición geopolítica y de significación histórica nacional; en este último caso basadas en la doctrina bolivariana y en la propia condición petrolera, han impulsado desde 1999 una manera de ejercer la diplomacia, de implementar una política exterior y de fundamentar una tesis sobre seguridad y defensa bastante singular, no sólo con respecto al período previo de la democracia representativa (1958 a 1999), sino también en forma comparada con el resto de las políticas exteriores de otros países de la región, inclusive de aquellos que se consideran aliados de Venezuela; tal es el caso de Cuba (CORRALES y ROMERO, 2013).

Las relaciones de Caracas con países que tienen una posición interna iliberal y una tendencia económica al estatismo, se constituyó por tanto en la prioridad de un diseño diplomático que ha contemplado no sólo la búsqueda de la reducción "al mínimo vital" de la dependencia de Venezuela de Estados Unidos, sino también la conformación de una estructura de poder que refleje un mundo multipolar y anti-capitalista.

Frente a la avalancha de este nuevo régimen, en el país ocurrieron dos situaciones no previstas. Por un parte, en junio de 2011 el presidente Chávez confirmó los rumores sobre su deteriorada salud y el comienzo de sus tratamientos que le redujeron su capacidad de dirigir el régimen. Y por la otra en ese mismo año, pero sobre todo a partir del año 2012 algunos economistas comenzaron a advertir sobre el advenimiento de un ciclo económico negativo para Venezuela, fundamentado en los datos que transmiten la 
reducción de los ingresos petroleros por la caída de los precios del barril a nivel mundial; por el estancamiento de la producción petrolera de Venezuela, del Producto Interno Bruto (PIB), de las reservas internacionales, del desenvolvimiento errático de la política cambiaria, del diferencial de precios entre los productos subsidiados y no subsidiados y por la generación de una escalada inflacionaria.

Esto se ha visto acompañado por un incremento exagerado de las importaciones de bienes y servicios, más del 50\% de ellas bajo el concepto de compras gubernamentales y por la caída vertiginosa de la industria privada, limitada esta en sus funciones por el control estatal y por el fracaso de los planes industriales y agrícolas del gobierno en las empresas nacionalizadas y en el experimento de las empresas de producción socialista y de las comunas. A esto se le añade el crecimiento exponencial de la economía informal, del contrabando fronterizo, de brotes de corrupción en la administración central y en el sistema de empresas estatales y en los programas sociales, entre ellos las misiones sociales.

En el marco de estos dos acontecimientos se celebraron las elecciones presidenciales de octubre de 2012 en donde compitieron el presidente Chávez ya en unas condiciones comprometidas por la enfermedad que lo aquejaba y el gobernador del estado Miranda, Henrique Capriles. Chávez resultó ganador con el 55.07\% y Capriles sacó el $44.1 \%$ de los votos en el contexto de una campaña oficialista orientada hacia la plataforma socialista y radical y la denuncia de la oposición sobre la conducta parcializada del Consejo Nacional Electoral.

\section{UNA TRANSICIÓN INESPERADA}

Hugo Chávez se dirigió al país por última vez y en su condición de presidente de la República el 8 de diciembre de 2012. En esa ocasión el líder del proceso bolivariano determinó que en caso de no irle bien en una nueva operación que se le tenía que practicar, asignaba la representación de su gobierno y del proceso chavista a Nicolás Maduro, el entonces Vicepresidente del Poder Ejecutivo. Imposibilitado de tomar posesión del cargo por cuarta vez y de acuerdo a una controversial decisión del Tribunal Supremo, se prolongó el mandato del presidente Chávez hasta su muerte, encargándose Nicolás Maduro desde ese momento de la presidencia de Venezuela hasta el 19 de abril de 2013, fecha en la cual se posesionó como presidente de la República para el período 2013-2019.

El Tribunal Supremo de Justicia dictaminó que el presidente Chávez no tenía que desincorporarse del cargo por la vía de la ausencia temporal o absoluta y que por lo tanto continuaba su gestión y que el juramento de ley para iniciar el nuevo período presidencial se podía postergar. De esta manera y bajo la tesis jurídica de la "continuidad administrativa" (por ser el mismo presidente saliente y entrante), el presidente Chávez continuó en su cargo, al igual que el Vicepresidente Nicolás Maduro. Esta decisión fue criticada por la oposición y definida por sus voceros como ilegal e ilegítima.

El presidente Chávez falleció el 5 de marzo de 2013 y por lo tanto el Vicepresidente Ejecutivo Nicolás Maduro se encargó de la Presidencia de la República Bolivariana de Venezuela. El Consejo Nacional Electoral convocó a elecciones presidenciales a fin de 
completar el periodo al cual había sido elegido Hugo Chávez en octubre de 2012. Con estos hechos se cerró otra etapa en la historia reciente del país.

El 14 de abril se dio la nueva elección presidencial, Nicolás Maduro ganó con una mínima diferencia, 50.61\% de los votos, seguido de Henrique Capriles con un 49.12\%, el candidato de la oposición quien obtuvo un sorprendente resultado. Nicolás Maduro se juramentó como nuevo presidente de Venezuela el 19 de abril de 2013, en medio de una fuerte crítica de la oposición que puso en duda los resultados electorales.

Quedan muchas cosas por discutir sobre el legado del presidente Chávez. Encabezó una revolución de tinte izquierdista en un país petrolero y consumista, en medio de un controversial entrenamiento de las masas en el credo socialista y la profesión de fe a un líder carismático que logró una proyección internacional y regional nunca vista en América latina y el Caribe desde los tiempos de Fidel Castro.

En segundo término, Chávez fue el líder fundamental de un grupo de políticos, intelectuales, militares, empresarios y dirigentes laborales y sociales que desplazaron a las élites tradicionales que habían gobernado al país desde 1958.

Y en tercer lugar, el presidente Chávez pudo mantener como jefe de Estado una serie de relaciones diplomáticas y comerciales con muchos países del mundo, varios de ellos sus socios y aliados, como Cuba, Irán, Siria, China y Rusia, con la mayoría de los países del orbe e inclusive mantuvo relaciones diplomáticas y comerciales con algunos gobiernos con quienes confrontó enormes problemas políticos, como lo fue Estados Unidos.

En cuanto al pasivo que Chávez dejó para la historia, cabe mencionar el alto costo que significó la polarización en Venezuela, la división ideológica y psicológica de sus habitantes, su hiper-presencia mediática y la tendencia a darle a la sociedad un tutelaje social que a la postre redujo las potencialidades de los venezolanos, al convertirlos fundamentalmente en meros recipientes de bienes y servicios subsidiados. No se puede dejar de lado su afanada tendencia a cercar a la empresa privada y a promover el estatismo con pocos resultados favorables y su poco apego por la institucionalidad.

Por otra parte, la delincuencia y los problemas de inseguridad siguen siendo problemas muy sentidos y sufridos por la población en todos sus estratos sociales. La mayoría de los indicadores internacionales sobre criminalidad dejan claro que Venezuela es uno de los países con mayor frecuencia de homicidios por habitante.

La Fuerza Armada Nacional Bolivariana, tal como se le conoce desde el año 2005 ha experimentado un profundo cambio como institución y en relación a su doctrina desde el año 1999, al dejar de ser una institución autónoma, para convertirse en el cuerpo armado del proceso chavista. De hecho, cuando el entonces presidente de Venezuela Hugo Chávez reiteró que sus fuerzas armadas eran chavistas, no estaba diciendo algo extraño. La organización militar se ha "colonizado" por un grupo de militares retirados y activos que están a favor de la revolución bolivariana. Es dentro de este contexto que la Fuerza Armada se convierte en un tema prioritario para el gobierno de Maduro, en el momento en que se genera una gran incertidumbre sobre la era post-Chávez. 
Cabe destacar cómo el régimen chavista ha avanzado en el control político y de inteligencia de la sociedad, pero no ha podido detener la crisis económica observada desde el año 2012. La caída de los precios del barril de petróleo, los resultados negativos del Producto Interno Bruto, la falta de reservas de divisas, la devaluación de facto y el aumento de precios de bienes y servicios, son la expresión más nítida de esa situación. Por otra parte, diversos sectores sociales claman por una mejoría de los sueldos y salarios y sigue aumentando el número de muertes violentas, destacándose el creciente número de asesinatos de funcionarios policiales.

Pero a pesar de una sensación de crisis generalizada, esta no se refleja en la pérdida de apoyo por parte del régimen y en una tendencia mayoritaria a favor de la oposición. ¿Por qué? Cuatro puntos son importantes: 1) El Gobierno sigue actuando como el principal proveedor de bienes y servicios; 2) La oposición luce dividida; 3) A la oposición le faltan recursos para garantizar el control de una votación que es manipulada por un ente electoral parcializado; 4) No hay ningún indicio de una intranquilidad militar. Por el contrario, se observa una creciente militarización del gobierno.

En otro orden de ideas, desde el punto de vista legislativo la llamada "Revolución Bolivariana" ha venido radicalizando su agenda intervencionista y estatista. Se trata de un paquete de leyes que comenzó a aprobarse en el 2007 y se aceleró en el 2010-2012 y en el 2013-2014 y que atentan directamente contra las libertades económicas frenando la producción y acorralando al sector privado de la economía.

La visión económica de la "revolución" se condensa en el modelo productivo socialista, tal y como reza en el Plan de Desarrollo Nacional Simón Bolívar (2007-13) y se observa en el Plan de la Nación para los años 2013-2019. Se desprende de estos textos que este control total se basa en la propiedad estatal de los factores de producción o en la regulación de los mismos. Un ejemplo claro es la Ley para la Defensa de las Personas en el Acceso de Bienes y Servicios que facilita la ocupación temporal de los factores de producción y coloca los derechos de propiedad en una situación de "concesión" del Estado venezolano.

En referencia al plano internacional, aparte de las manifestaciones de solidaridad de algunos Jefes de Estado y de gobierno con Venezuela en la ocasión de la enfermedad del presidente Chávez y de su posterior fallecimiento, el común denominador de las Cancillerías en la región, incluyendo al Departamento de Estado del gobierno de Estados Unidos, es el de aspirar a que esa transición "inesperada" originada por el fallecimiento del presidente Chávez fuera pacífica y bajo el marco constitucional. Los gobiernos extranjeros no querían que se diera un vacío político en el país por el hecho del fallecimiento del presidente Chávez ni que se dieran las condiciones para un régimen de facto en Venezuela (CORRALES y ROMERO, 2013).

Como una respuesta crítica a la globalización, se desarrolló una doctrina y una práctica anti-estatus heredada del Tercermundismo y de la cooperación Sur-Sur, pero que en el caso que nos ocupa ha contado con una plataforma más radical plasmada en todos los niveles, desde el tránsito de una política de alianzas extra-continental hasta el apoyo a partidos, organizaciones y personalidades de la izquierda mundial. Eso sí, Caracas ha contado para ello con recursos materiales y simbólicos (incluyendo el legado 
de Hugo Chávez) dentro de lo que significan los nuevos medios de comunicación, las llamadas redes y otros tipos novísimos de información (CORRALES y ROMERO, 2013).

El gobierno del presidente Maduro ha continuado y a su vez ha profundizado esta plataforma internacional, aumentando la frecuencia de los viajes presidenciales y diplomáticos, los acuerdos bilaterales y la potenciación de las organizaciones regionales como CELAC, UNASUR y ALBA, que a pesar de sus dificultades intrínsecas; falta de consenso, trabas para el comercio intra-regional y singularidades de cada una de las políticas exteriores de esos países, representan una aspiración a cambiar tanto el escenario internacional como el regional. En el caso de Venezuela, esto pasa por los esfuerzos para deslindarse de Estados Unidos y fomentar una proyección mundial y regional diferente (CORRALES y ROMERO, 2013).

Desde un punto de vista mundial, el presidente Maduro ha podido sortear la idea de que esta presencia de Venezuela se debió fundamentalmente al liderazgo carismático del fallecido presidente Chávez y a los recursos materiales provenientes de los ingresos petroleros. A casi tres años de su gestión esos obstáculos se han superado en parte.

La oposición venezolana ha destacado la necesaria relación política y económica con Estados Unidos y cómo el gobierno de Maduro se ha dedicado a agrietarla. Se tiene el caso de Guyana, en donde la política de solidaridad internacional del gobierno de Maduro (y de Chávez) han enfriado el reclamo que Venezuela tiene sobre una parte del territorio guyanés, tomando ventaja Georgetown en sus iniciativas para redimensionar las áreas territoriales terrestres y marinas y submarinas que están en discusión con Venezuela desde la firma del Acuerdo de Ginebra en 1966. Y se ha observado cómo el gobierno bolivariano ha tenido una serie de contratiempos con el gobierno de Colombia en relación a la política fronteriza de ambos actores.

En otras ocasiones, sectores críticos de la diplomacia venezolana contemporánea han puntualizado la ideologización del servicio exterior de Venezuela y su falta de profesionalismo, la injerencia de Cuba en los asuntos internos y externos de Venezuela y la innecesaria creación de conflictos políticos y diplomáticos con algunos gobiernos europeos como España, sin llegar al rompimiento de relaciones, como se ha dado de forma circunstancial en los casos de Colombia, Honduras, Panamá y Paraguay y en un caso más definitivo con Israel.

Otra consideración interesante se encuentra en el tema de la defensa del gobierno de Maduro frente a las ya cíclicas campañas diplomáticas y mediáticas en su contra, las cuales ha podido dominar: Se ha mantenido en el poder, ha neutralizado las iniciativas multilaterales para evitar la elección de Venezuela como miembro no permanente en el Consejo de Seguridad de Naciones Unidas, ha frenado las iniciativas de condenar la gestión del gobierno en la Organización de Estados Americanos (OEA) y la denuncia de sus prácticas comerciales en la Organización Mundial de Comercio (OMC), más otras iniciativas domésticas por parte de la oposición venezolana, como la de aspirar a que la comunidad internacional considere ilegítimo al gobierno de Maduro, a Venezuela como un Estado forajido o para promocionar el cambio de régimen en el país.

Lo cierto es que hasta la fecha, Venezuela ha podido mantener y profundizar la plataforma diplomática e internacional que se diseñó desde los primeros años de 
la revolución chavista, en el marco de una estructura internacional más compleja y menos antagónica, de transición hacia el multipolarismo, con un discurso predominantemente "progresista" y que en todo caso proporciona a Venezuela un papel menos costoso y menos negativo para quienes gobiernan el país, caso contrario cuando se trata de evaluar la crisis doméstica que tiene la nación (CORRALES y ROMERO, 2013).

Una consideración especial se merece la creciente preocupación de gobiernos, organismos multilaterales, organizaciones no gubernamentales y personalidades mundiales y regionales sobre el estado de los derechos humanos en el país, sobre la detención arbitraria de líderes y activistas políticos y sobre la inhabilitación de candidatos de la oposición postulados para las elecciones parlamentarias de diciembre de 2015. Todo esto ha llevado a que una buena parte de los actores internacionales comprometidos con el ejercicio democrático en el mundo vean con reservas la aplicación del estado de Derecho y el ejercicio democrático en Venezuela (MORSE, 2012).

Mientras tanto, Estados Unidos y la alianza occidental han mejorado sus relaciones con Irán y con Cuba, socios de Venezuela y con otros países simpatizantes de la causa revolucionaria. Sin embargo, las relaciones diplomáticas y comerciales entre Estados Unidos y nuestro país confrontan considerables obstáculos. El gobierno del presidente Maduro ha tenido que responder a los señalamientos, acusaciones, puntos de vista y reservas que diferentes actores de la vida estadounidense tienen sobre Venezuela, dentro del contexto de una crítica permanente de ellos sobre las limitaciones democráticas internas.

La posición del gobierno bolivariano sobre Estados Unidos se ha nutrido de la larga experiencia de los países que han tratado de desarrollar un política distante de los intereses de Washington. En el caso de Venezuela se ha ido más allá y en verdad, Venezuela ha sido un troublemaker de una importancia singular, algo que no se veía en América Latina y el Caribe desde el comienzo de la Revolución Cubana (CORRALES y ROMERO, 2013).

Venezuela presenta una severa crisis de gobernabilidad con el derrumbamiento económico, el crecimiento del crimen, la disfunción del sector público, la reducción de la producción petrolera, la demanda insaciable de dólares y la fuga de capitales. Esta crisis doméstica hace mucho daño a la capacidad del gobierno bolivariano de conducir una política exterior coherente. Como las ganancias de mantener un discurso antiimperialista radical parecen disminuir y la crisis de gobernabilidad del país se ha ampliado, Venezuela observa una conducta ambivalente.

Lo que sí es cierto es que Estados Unidos ha decidido llevar adelante una confrontación indirecta con Venezuela, creándole más problemas al gobierno de Maduro, pero de manera "sutil": atacando la supuesta vinculación de su liderazgo militar con el narcotráfico, apoyando a Guyana en su política de alterar lo estipulado en el Acuerdo de Ginebra y en general, en el asunto de la reclamación venezolana sobre parte del territorio de ese país, presionando a los gobiernos latinoamericanos para que se pronuncien sobre la violación de los derechos humanos en Venezuela y reduciendo las facilidades para que Venezuela pueda salir de esta enorme crisis económica. 
Durante el año 2014, se agudizó la crisis económica por el derrumbe de los precios petroleros, el déficit de la balanza de pagos, el peso de la deuda externa, la inflación y el crecimiento de la economía informal. Parte de la oposición promovió las manifestaciones callejeras y la protesta social, respondiendo el gobierno con una discutida acción represiva y judicial que en muchos casos puso en duda el talante democrático del régimen.

En el año 2015, el país presenta un balance poco optimista. A la caída de los precios del petróleo se le unen el alto costo de la vida, el auge de la delincuencia, la escasez de bienes y servicios y sobre todo la creciente crítica sobre el proyecto político que se inició en 1999. Esto también tiene su expresión internacional. El llamado socialismo del Siglo XXI ya no llama la atención, ni a los que temían su propagación continental ni a los que lo recibían con una emoción desmedida. La mayoría de las instituciones económicas de la región como el BID, el FMI, el BM y la CEPAL han pronosticado una reducción entre $-5 \%$ y $-9 \%$ del PIB para el año 2015.

Para el gobierno, su visión de la realidad se basa en la tradicional tesis conspirativa que de alguna forma achaca el origen de la crisis a una manipulación de Estados Unidos y sus aliados, a fin de reducir el poder de Rusia y de sus amigos, entre los cuales se encuentra Venezuela. La explicación favorita de la mayoría de los voceros de la oposición se basa en el impacto de la reducción de los precios del barril de petróleo como la principal causa de la crisis económica que vive el país.

Ambas posiciones no recogen con exactitud lo que está pasando. En verdad lo que se está viviendo es una crisis general de un modelo económico instaurado progresivamente y basado en un gasto público expansivo, en un control de cambio asfixiante, en la cruzada en contra del capital privado y en la generación de un movimiento consumista deformado, sea ya por el diferencial de precios, por las compras nerviosas o por la oportunidad de contrabandear bienes y servicios.

\section{CONCLUSIONES Y PROYECCIONES}

Con este artículo se aspira a dar una visión general sobre los últimos cincuenta y siete años de la vida republicana de Venezuela. Para ello se ha dividido la narrativa en dos momentos. Uno que va desde 1958, fecha del derrocamiento del gobierno de facto de Marcos Pérez Jiménez hasta la llegada de Hugo Chávez al poder en 1999. Y la otra que se desarrolla desde esa fecha hasta nuestros días.

En cuanto a la primera etapa conocida como el Sistema Populista de Conciliación de Élites o "puntofijismo", cabe destacar cómo los fundamentos que llevaron a ese sistema a perdurar por cuarenta años y a evitar su derrocamiento fueron cuestionados y remplazados por otros fundamentos que a su vez están siendo criticados por otro grupo de venezolanos. De esta manera se pudiera pensar que la discusión sobre historia reciente de Venezuela ha transcurrido entre los encuentros y desencuentros de al menos tres debates.

En primer término, me estoy refiriendo al consenso alcanzado por las élites que derrocaron a Pérez Jiménez y crearon el puntofijismo, el cual se contrapone al acuerdo alcanzado por Hugo Chávez y sus seguidores. El primero de ellos se basó en la negocia- 
ción política y en los pactos y acuerdos alcanzados bajo la idea de la mayoría relativa y la voluntad general. El segundo de ellos se basa en la tesis de la mayoría absoluta y la voluntad única, en la "oclocracia".

Un segundo concepto es el de la neutralidad de las Fuerzas Armadas. En el primer período estudiado, la no beligerancia del estamento militar en los asuntos políticos de la Nación fue una conditio sine qua non para la buena marcha del sistema. En el segundo período analizado, la politización de las Fuerzas Armadas y su beligerancia son consideradas como parte fundamental de la unión cívico-militar que gobierna desde 1999.

Un tercer concepto divergente entre las dos etapas es el del desarrollo económico. Para el primer período se consideró que con el capitalismo mixto y con la participación del Estado y de la empresa privada se lograría una sociedad equitativa. Durante el período que va desde el año 1999 hasta nuestros días, se considera que el estatismo y el socialismo son los instrumentos fundamentales del desarrollo, incluyendo los conceptos de la transferencia directa de los recursos materiales a la población, el tutelaje del Estado, la organización del pueblo a través de las comunas y desde luego, la concreción de un cerco legal e institucional a la iniciativa privada.

En definitiva, lo que contrasta es una visión política basada en la sociedad y en una visión política basada en el Estado como las palancas divergentes de dos propuestas sobre lo que debe ser un país. En función de esta consideración, cabe destacar la dificultad que tiene una sociedad polarizada, dentro de la contradicción permanente de un gobierno que aspira a profundizar un modelo basado en la idea de la democracia participativa y protagónica y en la oferta opositora de una nueva transición que aspira ir por el camino de la democracia liberal y la potenciación de la sociedad civil. De esta reflexión se origina una advertencia: que las propuestas intermedias del Estado social de derecho, de la democracia de partidos y del "reformismo" se reducen en cuanto a su praxis ante los planteamientos polarizantes de la profundización del régimen versus el cambio de régimen.

Desde luego que de esta discusión quedan algunos elementos importantes que considerar. En cada uno de estos modelos, se observa la contradicción permanente entre la aspiración de concretar un modelo de sociedad y un régimen político determinado y las limitaciones para llevar a cabo esos propósitos.

Una pregunta que pasa de un lado a otro es la referida a la cultura política y el apoyo popular a cada una de estas iniciativas. En la etapa del puntofijismo hubo la creencia generalizada que el venezolano había superado su visión paternalista y caudillista de la política y que habían logrado importantes avances en su comportamiento cívico y en su apego a la democracia. Es más, muchas de las críticas que recibe el Sistema de Conciliación de Elites están orientadas a plantear que éste fue el producto de un pacto por arriba y que no tomó en cuenta a las mayorías populares las cuales eran tan solo protagonistas en el momento electoral (PÉREZ SCHAEL, 1993; URBANEJA, 1991).

Ahora bien, el estudio minucioso de las encuestas de opinión de la época y de diversos estudios sobre cultura política que se administraron en esos años reportan, junto con los resultados electorales que obtuvieron los disidentes del sistema en los sucesivos 
procesos electorales, que no hubo una unanimidad, entre 1958 y 1999, en cuanto a los valores democráticos y la capacidad de aprendizaje del venezolano. Pero hay algo más interesante: tal como se demostró con el viraje de las mayorías hacia la oferta iliberal de Chávez, esa cultura democrática no se expandió y no permaneció en el tiempo tal como se hubiera querido ver (URBANEJA, 2000).

De igual forma, pero con un contenido diferente, la aspiración de la dirección política del nuevo régimen que se instaló progresivamente en Venezuela a partir de 1999, es la de implantar una cultura de izquierda revolucionaria fraguada en los moldes históricos del marxismo-leninismo que se ha manifestado en el uso del color rojo, en la polarización de la sociedad, en la propagación de la idea de la lucha de clases, del antagonismo social y de la sociedad tutelada bajo la tesis de la solidaridad comunal.

En ninguno de los dos casos, las cosmovisiones apuntadas se han completado, dada la existencia de unas prácticas sociales que de alguna manera han impedido su concreción. En primer término, la práctica social que cruza a ambas experiencias es la del populismo, entendido esta como la relación paternal entre el Estado y la sociedad que en el caso venezolano tiene como instrumento central el ingreso petrolero y como consecuencia de ello, la generación de una transferencia directa de recursos y una plataforma de pagos que va desde el tema salarial y los anexos laborales hasta la tolerancia de la corrupción administrativa, concebida esta como una forma de acumulación "originaria" capitalista (VERA, 2005),

A esta visión "economicista" utilizada por varios autores con el fin de explicar la lealtad al régimen (en los dos casos estudiados), se contrapone otra práctica social; tal es la del clientelismo político, basada en la militancia partidista y dentro de la concreción de un aparato ideológico y mediático en procura de un apoyo total al régimen. Si bien es cierto que en el período 1958-1999, esta política se basó en una vía indirecta y la nueva política a partir de 1999 en la vía directa - y sin muchas limitaciones -, ninguna de ellas evitó la presencia de un desarraigo político, lo que pudiera explicar, al menos en parte, el paso de una transición a otra.

Despejados estos problemas teóricos valdría la pena concluir este artículo con la presentación de algunos elementos coyunturales que a modo de conjetura caracterizan la situación actual en Venezuela. Desde el punto de vista político, cabe destacar que el país se mueve en torno a las elecciones parlamentarias del 6 de diciembre de 2015. El gobierno procura hacer útil su ventajismo al usufructuar y parcializar al sector público a su favor, como también la maquinaria partidista, la ingeniería electoral en cuanto a las inhabilitaciones de algunos candidatos de la oposición, la distribución de los cargos electorales, la puesta en duda de la pertinencia de una tarjeta unitaria de la oposición para participar en las elecciones parlamentarias y la continua descalificación de la oposición.

La oposición en tanto, observa el crecimiento de su opción electoral en las encuestas y mantiene una unidad precaria pero suficiente para contrarrestar lo que significa el peso asimétrico del poder que tienen el gobierno y el partido de gobierno, el PSUV.

Desde punto de vista económico, está a la vista que la situación de Venezuela es bastante paradójica. Por una parte, saltan los escándalos de corrupción administrativa, 
las alertas sobre que no hay dólares y la crítica a la política económica del gobierno. Pero por la otra, hay circulante en la calle, no hay indicios de la paralización de la economía, aunque sí un ajuste en materia de precios, en materia de impuestos y en materia de divisas. En este caso, hay que recordar que el gobierno no ha tomado una decisión sobre temas cruciales como lo son el aumento de la gasolina, la unificación del precio del dólar y el recorte de los gastos de PDVSA.

Frente a este panorama surge la duda sobre cómo está y cómo se proyecta a futuro la economía venezolana. En verdad, ella es el resultado de varios sectores que funcionan al mismo tiempo: el subsidiado, el de la economía del dólar y el de la economía "negra". Además de esas dimensiones está la economía tradicional, la cual depende del gasto público y de las exportaciones e importaciones legales. En materia de exportaciones lo que reporta la situación actual es la reducción por más de un 50\% de las ventas petroleras, dada la caída de los precios mundiales del barril de petróleo venezolano. En materia de importaciones, estas han bajado un 30\%, si se compara con los resultados del año 2012, aunque hay que tomar en cuenta que ese año fue considerado por los expertos como "anormal".

Desde la óptica de la dinámica social, uno de los más importantes problemas que tiene Venezuela es el referido a la ausencia del orden público. El Estado de Derecho, las instituciones dedicadas al tema de la seguridad y el accidentado mundo penal son algunos elementos que por su estado de descomposición actual, no garantizan que se pueda llevar adelante una política pública eficaz y eficiente que reduzca la indefensión del ciudadano frente a la violencia social y la violencia de Estado.

En efecto, "los partes" diarios sobre hechos contrarios a lo jurídico, la interpretación por la libre de las leyes y reglamentos y las presiones cotidianas sobre las organizaciones dedicadas a la denuncia de las violaciones de los derechos humanos, nos demuestran cómo se ha agigantado la distancia entre el derecho a la seguridad de personas y bienes y la asfixiante realidad delictiva, corrupta e informal. El toque de queda "de hecho" después de las ocho de la noche, la inseguridad para transitar por las calles y avenidas, el innovador robo desde una moto, el asalto a plena luz del día, la dificultad para conseguir bienes y servicios, la peligrosa desviación de la función pública y el desconocimiento del debido proceso, son apenas algunos puntos que llaman la atención.

El país presenta un balance poco optimista. A la caída de los precios del petróleo se le une el alto costo de la vida, el auge de la delincuencia y la escasez de bienes y servicios. Y el país de los humildes, el de la clase media empobrecida, el de los obreros, campesinos y excluidos se empieza a dar cuenta que su vida depende de la casualidad y del temperamento de alguien. Y otros piensan si es pertinente emigrar. Pero ni esto ni aquello es una condición suficiente para plantear que el país ya está colapsado, aunque pudiera estar dirigiéndose hacia esa dirección si no se corrigen los males señalados.

\section{BIBLIOGRAFÍA}

ÁLVAREZ, Ángel. (Coord.). El Sistema Político Venezolano: Crisis y Transformaciones. Caracas: Instituto de Estudios Políticos, UCV. 1997. 
ÁlVAREZ, Ángel. La Democracia Delegativa y Muerte de la Constitución. En: Fundación Manuel García Pelayo. p. 743-759. Caracas: Fundación Manuel García Pelayo, 2000.

BAPTISTA, Asdrúbal. Teoría Económica del Capitalismo Rentista. Economía, Petróleo, Renta. Caracas Ediciones IESA, 1997.

BLANCO, Carlos. Venezuela, Del Siglo XX al Siglo XXI: un Proyecto para Construirla. Caracas: Editorial Nueva Sociedad, 1993.

BOND, Robert. Contemporary Venezuela and its Role in International Affairs. New York: New York University Press, 1977.

CARDOZO DE DA SILVA, Elsa. Cuarenta años después: la política exterior que tuvimos y la que necesitamos. En: Revista Venezolana de Análisis de Coyuntura. v. IV, n. 1, p. 43-63, ene-jun. Caracas: Facultad de Ciencias Económicas y Sociales (FACES), Universidad Central de Venezuela, 1998.

CARRERA DAMAS, Germán. Historia Contemporánea de Venezuela. Bases Metodológicas. Caracas: Universidad Central de Venezuela, Ediciones de la Biblioteca, 1977.

CHALIAND, Gérard. Les Guerres Irréguliérs. Paris : Gallimard, 2008.

CHILCOTE, Ronald H. Revolution and Structural Change in Latin America. A Bibliography on Ideology, Development and the Radical Left (1930-1965). Stanford: Stanford University Press, v. 2, 1970. COPPEDGE, Michael. Venezuela: soberanía popular versus democracia liberal. En: Jorge Domínguez y Michael Shifter (Eds). Construcción de Gobernabilidad Democrática en América Latina, p. 183212. México: Fondo de Cultura Económica, 2005.

CORRALES, Javier y ROMERO, Carlos A. U.S.-Venezuela Relations since the 1990's: Coping with Mid-Level Security Threats. En: Contemporary Inter-American Relations Series. New York: Routledge Press, 2013.

CORRALES, Javier and PENFOLD, Michael. Dragon in the Tropics. Hugo Chávez and the political economy of revolution in Venezuela. Washington, DC: The Brookings Institution Press, 2011.

CORRALES, Javier. Hugo Boss. How Chavez is refashioning dictatorship for a democratic age. En: Foreign Policy, jan-feb, p. 32-40. Washington: Carnegie Endowment for International Peace, 2006.

DIAMINT, Rut. Cuestiones Militares en América Latina. En: Jorge Domínguez y Michael Shifter (Eds). Construcción de Gobernabilidad Democrática en América Latina, p. 47-81. México: Fondo de Cultura Económica, 2005.

DIETZ, Henry and MYERS, David. From Thaw to Deluge: Party System Collapse in Venezuela and Peru. En: Latin American Politics and Society, v. 49, n. 2, p. 61-70. Miami: University of Miami, Center for Latin American Studies, 2007.

DUNNING, Thad. Crude Democracy: Natural Resource Wealth and Political Regimes. New Haven: Yale University Press, 2008.

ELLNER, Steve. Recent Venezuelan Political Studies: A Return to Third World Realities. En: Latin American Research Review. v. 32, n. 2, p. 201-218. Pittsburgh: Latin American Studies Association, 1997.

GÓMEZ CALCAÑO, Luis; PATRUYO, Tharalí. Entre la Esperanza Popular y la Crisis Económica. Transición Política en Venezuela. En: Cuadernos del CENDES. Dossier: Globalización, Reestructuración y Transformación Territorial, ene-abr, p. 199-246. Caracas: CENDES, Universidad Central de Venezuela, 2000.

HACHEMAOUI, Mohammed. La rente entrave-t-elle vraiment la démocratie? Réexamen critique des théories de «l'État rentier » et de la «malédiction des ressources.. En : Revue française de science politique v. 62, n. 2, abr, p. 207-230. Paris: Institute du Science Politique, 2012.

KARL, Terry Lynn. The Paradox of Plenty. Oil Booms and Petro-States. Berkeley: University of California Press, 1997 
KORNBLITH, Miriam. Venezuela. De la Democracia Representativa al Socialismo del Siglo XXI. Borrador, Caracas: Instituto de Estudios Políticos, Universidad Central de Venezuela, 2007.

KORNBLITH, Miriam. Crisis y transformación del sistema político: nuevas reglas de juego. En: Álvarez, Ángel (Coord). El sistema político venezolano: crisis y transformaciones, p. 1-31. Caracas: Instituto de Estudios Políticos, Universidad Central de Venezuela, 1997.

LEVINE, Daniel. Conflict and Political Change in Venezuela. Princeton: Princeton University Press, 1973.

LINDBERG, Staffan. Democratization by Elections: A New Mode of Transition. Baltimore: Johns Hopkins University Press, 2009.

MAINWARING, Scott. From Representative Democracy to Participatory Competitive Authoritarianism: Hugo Chávez and Venezuelan Politics. En: APSA. Perspectives on Politics. dec.- 2012, v.10, n. 4, p. 955-967. Washington: APSA, 2012.

MARTÍNEZ MEUCCI, Miguel. Apaciguamiento. El Referéndum Revocatorio y la consolidación de la Revolución Bolivariana. Caracas: Alfa Editores, 2012.

MARTZ, John y MYERS, David J. Venezuela: the Democratic Experience. New York: Preger Publishers, 1977.

MC COY, Jennifer y MYERS, David J. The Unraveling of Representative Democracy in Venezuela. Baltimore: The John Hopkins University Press, 2004.

MORGAN,Jana. Bankrupt Representation and Party System Collapse. Pennsylvania: The Pennsylvania State University Press, 2011.

MORSE, Yonatan L. The Era of Electoral Authoritarianism. Review article. En: World Politics, jan. 2012, p. 161-198. Princeton: Princeton Institute for International and Regional Studies, 2012.

NAIM, Moisés y PIÑANGO, Ramón. El Caso Venezuela. Una Ilusión de Armonía. Caracas: IESA, 1984. PÉREZ SCHAEL, María Sol. Petróleo, Cultura y Poder en Venezuela. Caracas: Monte Ávila Editores Latinoamericana, 1993.

REY, Juan Carlos. La Democracia Venezolana y la Crisis del Sistema Populista de Conciliación. Madrid: Centro de Estudios Constitucionales, 1991.

REY, Juan Carlos. El Futuro de la Democracia en Venezuela. Serie Estudios. Caracas: IDEA, 1989.

REY, Juan Carlos. El Sistema de Partidos Venezolano. En: POLITEIA, n. 1. Caracas, Instituto de Estudios Políticos, Universidad Central de Venezuela. p. 175-230,1972.

ROMERO, Aníbal. La Miseria del Populismo. Caracas: Editorial Centauro, 1986.

ROMERO, Carlos A. Jugando con el Globo. La política exterior de Hugo Chávez. Caracas: Ediciones B, 2006.

ROMERO, Carlos A. Cuba y Venezuela. La génesis y el desarrollo de una utopía bilateral. En: Luis Fernando Ayerbe (Coord.). Cuba, Estados Unidos y América Latina frente a los desafíos hemisféricos, p. 159-202. Barcelona, España: Icaria Editorial, IEEI-UNSP, CRIES, 2011.

ROSS, Michael. Does Oil Hinder Democracy? En: World Politics. v. 53 n. 3, p. 325-61. Princeton: Princeton Institute for International and Regional Studies, 2011.

SILVA MICHELENA, José Agustín. Exploraciones en Análisisy Síntesis. Caracas: Universidad Central de Venezuela, 1967.

SMILDE David y HELLINGER, Daniel. Venezuela's Bolivarian Democracy Participation, Politics, and Culture under Chávez. Durham, NC: Duke University Press, 2011.

URBANEJA, Diego Bautista. Pueblo y Petróleo en la Política Venezolana del Siglo XX. Caracas: Centro de Formación y Adiestramiento de Petróleos de Venezuela y sus Filiales, 1991. 
URBANEJA, Diego. El Proceso Constituyente Venezolano de 1999 y el Concepto de lo Político de Carl Schmitt. En: Fundación Manuel García Pelayo, p. 761-781. Caracas: Fundación Manuel García Pelayo, 2000.

VERA, Leonardo. Gobernabilidad y Estado Rentístico: el valor estratégico interno del recurso Petrolero. En: María Ramírez Ribes (Compilación y Prólogo), Gobernanza, laberinto de la democracia, p. 255256. Caracas: Club de Roma, 2005.

WEYLAND, Kurt. The Rise of Latin America's Two Lefts: Insights from Rentier State Theory. En: Comparative Politics, v. 41 n. 2, p. 145-164. New York: Graduate Center, CUNY, 2009.

WEYLAND, Karl. Economic Voting Reconsidered: Crisis and Charisma in the Election of Hugo Chávez. En: Comparative Political Studies. n. 36. Washington: SAGE, sept., n. 7 p. 822-848, 2003. 